\title{
MOBBING ALS MÖGLICHE URSACHE FÜR BRUXISMUS
}

Folgen von Stress-- Nächtliches Zähneknirschen bei Teenagern könnte laut einer brasilianischen Studie an Mobbing liegen. Wie die Dental Tribune schreibt, wurden in einer Fall-Kontroll-Studie 309 Jugendliche im Alter zwischen 13 und 15 Jahren untersucht. Die Fallgruppe bestand aus 103 Probanden, die von schlafbezogenem Bruxismus berichteten, und 206 Probanden in der Kontrollgruppe, bei denen nächtliches Zähneknirschen ausgeschlossen werden konnte. Stress gilt schon lange als eine der Ursachen für nächtlichen Bruxismus. Auch die Ergebnisse des brasilianischen Forscherteams belegen diesen Zusammenhang. 134 Probanden
(43,3 Prozent der Befragten), davon sind 90,3 Prozent männlich, gaben an, schon einmal mit verbalem Mobbing in der Schule konfrontiert worden zu sein, sowohl in der Rolle als Täter, Opfer oder beides. 65 Prozent der MobbingErfahrenen litten unter nächtlichem Zähneknirschen, bei den Teilnehmern ohne Mobbing-Erfahrung knirschten nur 17 Prozent nachts mit den Zähnen.

Damit sind Jugendliche, die unter Bruxismus leiden, sechs Mal häufiger in Mobbing involviert als Teenager, die nicht knirschen. RED

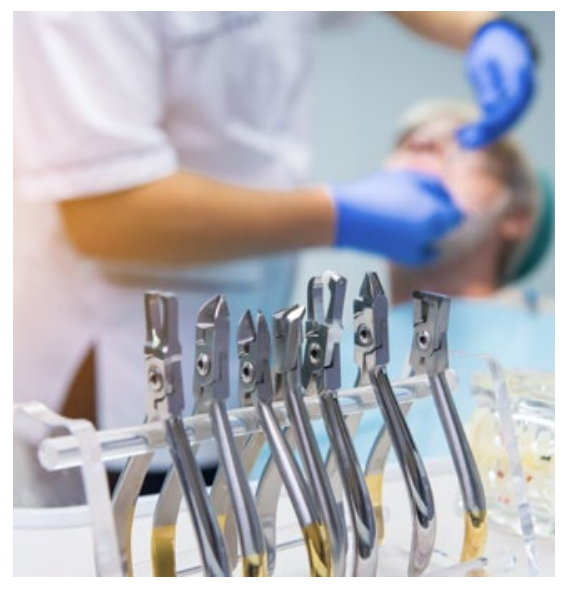

\section{WISSEN KOMPAKT}

Fortbildung im November-- Kieferorthopädie und MKG-Chirurgie weisen einige Schnittstellen auf - zum Beispiel beim Einsatz skelettaler Verankerungen, um unerwünschte Nebeneffekte und Zahnbewegungen während einer kieferorthopädischen Behandlung zu vermeiden. Auch bei skelettalen Dysgnathien hat sich die kombinierte kieferorthopädischkieferchirurgische Therapie etabliert. Außerdem scheint die chirurgische Kortikotomie im Rahmen der kieferorthopädischen Behandlung Erwachsener zu einer beschleunigten Zahnbewegung und entsprechend verkürzten Behandlungsdauer zu führen.
Daher geht es in der November-Ausgabe von wissen kompakt in drei kompakten Fortbildungsbeiträgen um das interdisziplinäre Thema „Kieferorthopädie an der Schnittstelle zur MKG-Chirurgie“:

\section{„KORTIKOTOMIE - AKZELERIERTE KIEFER- ORTHOPÄDIE"}

von Dr. S. Schramm, Dr. U. Schulze-

Späte, PD Dr. M. Wolf

\section{„VERLUSTRATEN VON GAUMENIMPLAN- TATEN UNTER ANWENDUNG VON WUND- HEILPLATTEN"}

von Dr. S. Chhatwani, B. Chhatwani B.D.S, Dr. P. Gebhardt, Dr. E. Johannsen, PD Dr. Dr. C. Jacobs

\section{„DYSGNATHIECHIRURGIE KOMPAKT"}

von PD. Dr. Dr. M. Moergel , Dr. S. Wriedt

Die Teilnahme an den ContinuingMedical-Education(CME)-Fortbildungen erfolgt online auf www.SpringerZahnmedizin.de und ist für die Mitglieder des Freien Verbandes Deutscher Zahnärzte (FVDZ) sowie für Abonnenten von wissen kompakt kostenlos. Beides trifft nicht auf Sie zu? Die entsprechenden Anträge finden Sie in dieser Ausgabe ab Seite 59.

wissen kompakt - gebündelte zertifizierte zahnärztliche Fortbildung

\section{HERZLICHEN GLÜCKWUNSCH!}

Preisausschreiben-- Dr. Jean-Arno Topp aus München ist der Gewinner unseres Kreuzworträtsels aus der DFZ-Sommerausgabe. Er habe sich sehr gefreut, sagte das FVDZ-Mitglied der Redaktion am Telefon: „Der Gewinn ist eine tolle Sache. Ich mache immer alles mit, was sich an Preisausschreiben bietet." Den 200-Euro-Gutschein für ein Seminar der FVDZ akademie werde er wohl in eine Fortbildung zum Thema Praxismanagement investieren. MAR

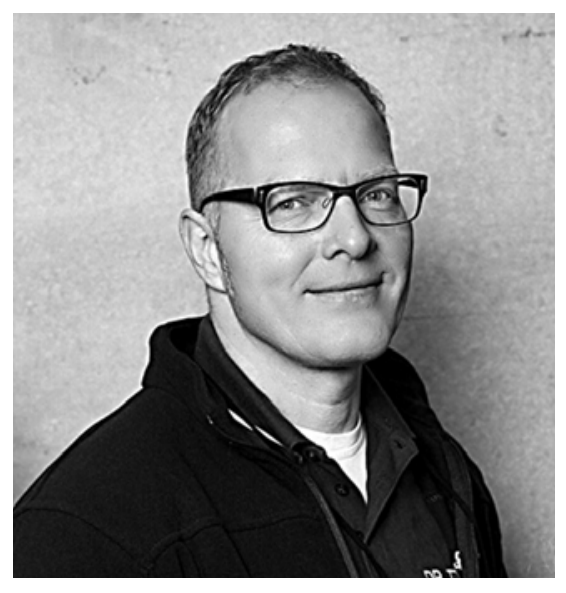

